\title{
How psychological frustration tolerance and self- esteem influence the association between father absence and hostility in depressed adolescents: a multiple mediation model
}

\section{Xiao-Ge Liu}

China University of Geosciences

\section{Ru-Yue Xie}

China University of Geosciences

\section{Yang Li}

Zhengzhou Industry and Trade School

\section{Fang Xiong}

China University of Geosciences

Wen-Tian Li ( $\square$ liwentian2020@126.com )

Wuhan Mental Hospital, University of Science \& Technology

\section{Research Article}

Keywords: Depressed adolescents, Hostility, Father absence, Psychological frustration tolerance, Selfesteem

Posted Date: March 16th, 2021

DOl: https://doi.org/10.21203/rs.3.rs-289958/v1

License: (c) (i) This work is licensed under a Creative Commons Attribution 4.0 International License. Read Full License 


\section{Abstract}

Background: In China, father absence is a very common phenomenon, causing many mental health problems, such as the hostility of depressed teenagers. This study is to explore the relationship between father absence and hostility of depressed adolescents as well as the mediating effects of self-esteem and psychological frustration tolerance.

Methods: We conducted a cross-sectional study among depressed adolescents in Wuhan mental health center. They were assessed with Father absence questionnaire, Chinese Hostility Inventory, Psychological Endurance Questionnaire and Self-esteem Scale. We used PROCESS macro for SPSS to perform mediation analyses.

Results: The level of adolescent hostility was positively correlated with father absence, and negatively correlated with self-esteem and psychological frustration tolerance. Father absence had not a direct impact on the level of hostility with depression, but had indirect impact via psychological frustration tolerance and self-esteem.

Conclusion: Psychological frustration tolerance and self-esteem has a chain mediating effect on the relationship between father absence and hostility. Future intervention strategies could focus on psychological frustration tolerance and self-esteem to reduce the level of hostility of adolescents with depression.

\section{Background}

Adolescence is an important period of growth in a person's life. Studies have shown it is a period of high incidence of mental illness [1], such as depression. According to statistics on the cause of death among adolescents aged 15-19, depression has become the second leading cause of death [2]. In recent years, it has been reported frequently that young people hurt themselves and others. The generally high level of hostility among adolescents may be an important factor. The detection rate of adolescents' highly aggressive behavior has reached $26.1 \%$. [3]. Moreover, the level of hostility of adolescents with depression is often higher than that of ordinary adolescents. Because of the high level of hostility, many adolescent patients are poor compliance with treatment, which not only brings difficulties to treatment, but also wastes medical resources, causing a vicious circle. However, there were few researches on hostility in China, and there was no empirical research that could be used for reference. Therefore, this paper studied the hostility of depressed teenagers in order to enrich the research results related to hostility.

In 2000, Lin Yimei referred to hostility as verbal attack, physical attack, and suppressing hostility without expressing it.

Hostility will destroy teenagers' physical and mental health, interpersonal relationship and family harmony, and bring serious adverse effects to families and society [4]. It has been clinically proven that the severer the patient's depressive symptoms, the higher his level of hostility [5]. The well-known 
psychiatrist Karen Horney mentioned in her book "The Neurotic Personality of Our Time" that children will feel insecure if they do not receive or lack the real care from their parents in childhood, which will trigger basic hostility [6]. Under the influence of the traditional Chinese culture of "the male dominates outside", the father absence is a very common phenomenon: a study found that in the semi-stay state (only one parent goes out for working, while the other one stays at home), the percentage of families with the father out of town was $82.82 \%$ [7]. Father absence refers to the phenomenon that children lack father's care and love due to various reasons [8]. In addition to the father is dead or does not live with his children, it also includes the father's lack of functionality caused by his busy father or poor communication between father and son (psychological deficiency) [9]. And studies have shown that father absence was closely related to the level of hostility of depressed teenagers [10][11]. So, how does father absence cause hostility? This study focused on the depressed adolescent population and explored the relationship between father absence and the hostility of adolescents.

Self-esteem is an individual's evaluation of self-worth, perception of self-capability, and acceptance of the overall self [12]. Self-esteem stems from the individual's early experience and will remain relatively stable after formation [13]. Abundant evidence has shown that teenagers with father passing away often have the problem of low self-esteem level [14][15]. There is a significant negative correlation between selfesteem and aggressive behavior [16] [17], and a large number of studies have proved that aggressive behavior is positively related to hostility [18] [19], which infers that self-esteem and hostility are also negatively correlated. Self-esteem is an important psychological dimension of adolescents. Depressed adolescents often have the lower level of self-esteem, and self-esteem has an important impact on the hostility of adolescents.

Psychological frustration tolerance is the ability to overcome obstacles and withstand stressful situations [20]. It is the ability to tolerate setbacks and adopt a positive mental mechanism when experiencing adversity, trauma or major negative events. According to Berkowitz's frustration-aggression hypothesis and the viewpoint of new cognitive connectionism, hostility is caused when an individual feel frustrated [21], and some studies have shown that hostility is significantly negatively correlated with psychological frustration tolerance [22], while many individual personality characteristics and will-power qualities, such as decisiveness, tenacity, courage, optimism and self-confidence are all related to father's upbringing [23]. Therefore, it can be assumed that when the father is absent, the teenager feels more frustrated facing with setback, which will produce a stronger anger and hostility towards others or themselves.

Although both self-esteem and psychological frustration tolerance may be mediating variables between father absence and adolescent hostility level, there is a certain correlation between them, instead of parallel relationship. A previous study has shown that the lower the level of self-esteem of an individual, the lower the psychological frustration tolerance [24], and that people with high self-esteem have better psychological adaptability, and can regulate their mood and behavior better [25], so they can cope with setbacks better. 
This study aim was to determine potential mechanisms underlying the relationship between father absence and the level of hostility in depressed adolescents. Specifically, we will examine how father absence influences hostility through self-esteem and psychological frustration tolerance. Based on the literature review, we propose the following three hypotheses:

\section{Hypothesis 1}

Self-esteem is a mediating variable between the degree of father absence and the level of hostility.

\section{Hypothesis 2}

psychological frustration tolerance is a mediating variable between the degree of father absence and the level of hostility.

\section{Hypothesis 3}

father absence affects the level of hostility in depressed teenagers through multiple mediating roles of self-esteem and psychological frustration tolerance.

\section{Methods}

\section{Participants}

All participants were recruited from Wuhan mental health center located in Hubei region in China. A questionnaire survey was conducted in adolescent depressed inpatients and outpatients. The inclusion criteria were: (1) ICD-10 diagnosis of depression, diagnosed by two physicians with the title of deputy director of psychiatry or above; (2) Aged 12 to 25 years old (the age boundary standard refers to the international legal age [26], previous researches [27] and the characteristics of the development of adolescents' self-concepts [28]); (3) Ability to understand the survey instructions and willingness to provide written informed consent.

The exclusion criteria were as follows: coexisting mental retardation, dementia, or other severe organic disorders; drug or alcohol abuse; schizophrenia; patients who currently suffer from severe physical diseases thus unavailable for participation. A total of 137 cases met the above criteria, including 56 males and 81 females; their age was $(18.41 \pm 3.69)$ years, and the degree of father absence was (3.68 \pm 1.13).

Before the investigation, necessary communication with the directors and head nurses in charge of each ward was required for cooperation. For all potential participants, inform them and their families of the purpose of the questionnaire, and the principle of voluntary participation. The questionnaire filled in is only used for scientific research with protection for patient privacy.

Tools 
Father absence measured by the questionnaire developed by Zhu Shuzhen [29].

The instrument consists of 10 items, which describe the existence of father for the participants to choose based on their own conditions.

The father's living condition at home (physiological deficiency) combined with the father-son relationship level (psychological deficiency) is taken as the test criteria of father absence. The higher the scores of these two items, the more serious the father absence is, with the lowest score as 2 points and the highest score as 7 points.

\section{Chinese Hostility Inventory (CHI)}

$\mathrm{CHI}$ was Compiled by Taiwan scholar Lin Yimei [30]. This is a self-report questionnaire with 20 items, which rates on a 5-point Likert scale. It contains four subscales: hostile cognition, hostile emotion, expression of hostility, and suppression of hostility. Among them, the subscales of hostile cognition, hostile emotions, and expression of hostility are revised from the "Attack Questionnaire". The suppression of hostility subscale is added in consideration of the characteristics of Chinese culture. That subscale is adapted from the subscale of "anger in" (which means holding anger inside without expression) in "Anger Expression Scale (AX)" [31][32]. Higher scores indicate higher levels of hostility.

\section{Psychological Frustration Tolerance Questionnaire}

The questionnaire was compiled by Xie Bingqing and Wu Ziqiang [33]. It includes 30 items in total, and the answers are "yes" and "no". The answer "yes" is counted as 1 point, and "no" is counted as 0 point. Part of the negatively expressed items are reversely scored. The higher the total score, the higher the participant's psychological frustration tolerance.

\section{Self-esteem Scale (SES)}

The SES compiled by Rosenberg (1965) [34] was used for the survey. The questionnaire is a singledimensional questionnaire consisting of 10 items, in which questions $3,5,8,9$, and 10 are reversely scored. The questionnaire rates on a 4-point scale ( 1 as totally inconsistent while 4 as completely consistent). Higher scores indicate higher levels of self-esteem.

\section{Statistical analysis}

SPSS 21.0 was used for data cleaning, coding, and preliminary analysis. Spearman correlation was used to evaluate the correlations between variables. The mediation model was analyzed using the PROCESS macro for SPSS. All continuous variables were standardized. In this study, we bootstrapped 5000 samples from the data, and $95 \%$ bootstrap confidence intervals $(\mathrm{Cl})$ were calculated.

\section{Results}

\section{Common method deviation test}


Using Harman's single factor test, the results showed that there were 18 factors with characteristic roots greater than 1 , and the variance explained by the first factor was $20.61 \%$, which was less than the critical value of $40 \%$ [35], so there was no obvious common method deviation in this study.

\section{Correlational Analysis}

Correlation analysis showed that the degree of father absence was significantly negatively correlated with self-esteem and psychological frustration tolerance $(p<0.001)$, and was significantly positively correlated with hostility level $(p<0.001)$. Self-esteem was significantly positively correlated with psychological frustration tolerance $(p<0.001)$. Hostility level was negatively correlated with self-esteem and psychological frustration tolerance $(p<0.001)$. More information about results of correlation analysis is provided in Table 1.

Table 1

Descriptive Statistics and Correlation of Variables

\begin{tabular}{|lllll|}
\hline & 1 & 2 & 3 & 4 \\
\hline 1. Father absence & & & & \\
\hline 2. Self-esteem & & & & \\
\hline 3.Psychological frustration tolerance & $-0.34^{\star \star \star}$ & $0.74^{\star \star \star}$ & & \\
\hline 4. Hostility & $0.30^{\star \star \star}$ & $-0.64^{\star \star \star}$ & $-0.69 * \star \star$ & \\
\hline $\mathrm{M} \pm \mathrm{SD}$ & $3.68 \pm 1.13$ & $24.10 \pm 6.34$ & $13.12 \pm 6.48$ & $63.41 \pm 14.26$ \\
\hline Note: ${ }^{*} P<0.05,{ }^{* * P<0.01, * \star * P<0.001, ~ t h e ~ s a m e ~ b e l o w . ~}$ & & \\
\hline
\end{tabular}

\section{The relationship between the degree of father absence and the level of hostility in depressed adolescents: a chain mediation effect test}

According to the results of correlation analysis, self-esteem and psychological frustration tolerance meet the statistical requirements for further testing of mediation effects [36]. Taking the degree of father absence as the independent variable, hostility level as the dependent variable, self-esteem and psychological frustration tolerance as the mediating variables, a multi-level regression analysis was carried out according to Model 6 in the PROCESS macro for SPSS, which can be used for an integrated test of the chain mediation model [37]. The gender was taken as a control variable.

Regression analysis showed (Table 2): the direct predictive effect of father absence on the level of adolescent hostility was not significant $(\beta=0.07, p>0.05)$, but it significantly positively predicted the level of adolescent hostility on the whole $(\beta=0.26, p<0.01)$. Father absence directly negatively predicted the level of self-esteem $(\beta=-0.22, p<0.01)$, and negatively predicted psychological frustration tolerance $(\beta=-0.19, P<0.01)$. Self-esteem directly predicted psychological frustration tolerance directly $(\beta=0.67, p<$ 
0.001). Self-esteem and psychological frustration endurance tolerance negatively predicted the level of adolescent hostility $(\beta=-0.26, p<0.01 ; \beta=-0.45, p<0.001)$.

Table 2

Regression analysis of the model between absence of father and hostility level

\begin{tabular}{|c|c|c|c|c|c|c|}
\hline & \multicolumn{2}{|c|}{ Self-esteem } & \multicolumn{2}{|c|}{ Psychological frustration tolerance } & \multicolumn{2}{|c|}{ Hostility } \\
\hline & $\beta$ & $t$ & $\beta$ & $t$ & $\beta$ & $t$ \\
\hline Gender & -0.24 & $-2.99 * *$ & -0.10 & -1.71 & 0.07 & 1.01 \\
\hline Father's absence & -0.22 & $-2.63^{\star \star}$ & -0.16 & $-2.67^{\star \star}$ & 0.07 & 1.03 \\
\hline Self-esteem & & & 0.67 & $11.18^{* * *}$ & -0.26 & $-2.92^{\star \star}$ \\
\hline Frustration tolerance & & & & & -0.45 & 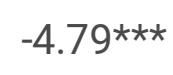 \\
\hline$R$ & 0.35 & & 0.76 & & 0.72 & \\
\hline$R^{2}$ & 0.12 & & 0.58 & & 0.51 & \\
\hline$F$ & $9.42 * *$ & & $61.29 * * \star$ & & 34.85 & \\
\hline
\end{tabular}

The analysis results of the mediation effect showed (Table 3 and Fig. 1): self-esteem and psychological frustration tolerance had a completely mediating effect on the relationship between father absence and the level of adolescent hostility, and the mediation effect value was 0.19 , accounting for $73.08 \%$ of the total effect of father absence on hostility level. Specifically, the mediating effect consisted of indirect effects produced through three paths: the indirect effect 1 (0.06): father absence $\rightarrow$ self-esteem $\rightarrow$ hostility level; the indirect effect 2 (0.07): father absence $\rightarrow$ psychological frustration tolerance $\rightarrow$ hostility level; the Indirect effect 3(0.06): father absence $\rightarrow$ self-esteem $\rightarrow$ psychological frustration tolerance $\rightarrow$ hostility level. The data in Table 3 showed that the three indirect effects accounted for $23.08 \%, 26.92 \%$ and $23.08 \%$ of the total effect respectively. The Bootstrap $95 \%$ confidence intervals didn't contain a value of 0 , indicating that the three indirect effects were significant. 
Table 3

Bootstrap analysis of the mediation effect test

\begin{tabular}{|c|c|c|c|c|c|}
\hline \multirow{2}{*}{$\begin{array}{l}\text { Mediation } \\
\text { Path }\end{array}$} & \multirow{2}{*}{$\begin{array}{l}\text { Point } \\
\text { Estimate }\end{array}$} & \multirow{2}{*}{$\begin{array}{l}\text { Bootstrap } \\
\text { S.E. }\end{array}$} & \multicolumn{2}{|c|}{ BOOSTRAP 5000 TIMES 95\% Cl } & \multirow[t]{2}{*}{ Effect percentage } \\
\hline & & & Lower & Upper & \\
\hline Total indirect effect & 0.19 & 0.05 & 0.09 & 0.29 & $73.08 \%$ \\
\hline Ind 1 & 0.06 & 0.02 & 0.01 & 0.11 & $23.08 \%$ \\
\hline Ind 2 & 0.07 & 0.03 & 0.01 & 0.13 & $26.92 \%$ \\
\hline Ind 3 & 0.06 & 0.03 & 0.02 & 0.12 & $23.08 \%$ \\
\hline
\end{tabular}

\section{Discussion}

The results of this study showed that father absence didn't directly predict the hostility level of depressed adolescents, but it acted on hostility through mediation of self-esteem and psychological frustration tolerance respectively, and the chain mediation of those two factors. This is consistent with the results of existing studies. For example, it is found that family function has an impact on adolescents' hostility [38], while parents are the key to fulfilling family functions, and the absence of fathers will inevitably bring an impact. For example, studies have found that children who lack fatherly love are prone to tense relationship with their mothers during puberty, and they are disgusted with or hostile to their mothers [39].

Secondly, this study found that self-esteem played an important mediating role between father absence and the hostility level of depressed teenagers. The results showed that the higher the degree of father absence, the lower the level of self-esteem, which would predict the higher level of hostility. Previous studies have shown that father absence often leads to the problem of low self-esteem in adolescents [40]. In adolescence, the self-esteem of an individual is more derived from the evaluation of parents and others.

While in the family where the father is absent, because the father is not at home or cares less about the children, the children receive less encouragement or praise from the father, resulting in lower level of selfevaluation.

And lower self-evaluation makes it easier to internalize negative evaluation of the outside world, causing a lower level of self-esteem. Traditionally, it is believed that low self-esteem can easily lead to anger and aggressive behavior, which means that individuals with low self-esteem have a higher level of hostility. Low self-esteem will have a negative impact on an individual's social links, causing the individual to be inconsistent with social norms, and thus increasing hostile behaviors [41]. Rogers believes that if there is a lack of positive self-attention, individuals are more prone to hostility, resulting in aggressive behaviors 
[42]. That means if depressed adolescents get more companionship and communication from their fathers, they can have better self-evaluation, thereby reducing the level of hostility and the occurrence of psychological problems.

Thirdly, psychological frustration tolerance also played an important mediating role between father absence and hostility. The frustration-hostility theory believes that when a person's motivation or behavior is frustrated, it will produce offensive and aggressive reactions. Depressed adolescents whose fathers is missing have low level of psychological frustration tolerance and mental resilience due to lack of psychological resources from their fathers [43]. Compared with ordinary teenagers, they tend to experience more negative events and pressures, with the reaction of a strong sense of frustration, resulting in stronger anger and hostility.

Finally, the degree of father absence affected the hostility level of depressed teenagers through the chain mediating effect of self-esteem and psychological frustration tolerance. Depressed adolescents whose fathers is missing have lower self-esteem, while those with high self-esteem can better regulate their mood and behavior, have good psychological adaptability [25], can deal with frustration in a more active way, and have a higher level of endurance. Depressed teenagers with low self-esteem tend to adopt negative methods such as self-blame, withdrawal, and fantasies to cope with setbacks. They are easily defeated by setbacks, and thus become hostile.

\section{Limitations}

Because the present study was based on a self-reported questionnaire, there might be many associated confounding factors. In addition, a cross-sectional survey cannot be used to draw a definitive conclusion. Thus, further study requires a longitudinal design. In addition, due to the specific participant of the research, the sample size was not large, which could affect the generalizability of the findings, so it is necessary to increase the sample size in the later related research. Finally, besides the influence of selfesteem and psychological frustration tolerance, there must be other variables affecting the relationship between father absence and hostility which are in need for further discussion.

\section{Conclusion}

The present study verified the mediating effect of psychological frustration tolerance and self-esteem on the relationship between father absence and hostility. Our results helped to reveal the underlying influencing mechanisms between these psychological variables (father absence, psychological frustration tolerance, self-esteem) and hostility. The research inspired us to pay attention to the impact of father absence on depressed teenagers, to improve their self-esteem, cultivate psychological frustration endurance, reduce hostility and improve mental health. Confirmation of these results may be helpful to develop targeted interventions to reduce the adolescent hostility.

\section{Abbreviations}


CHI: Short Form Chinese Hostility Inventory; SES: Self-esteem Scale; Cl: Confidence intervals

\section{Declarations}

\section{Acknowledgments}

We are especially grateful to the individuals who volunteered their time to participate in this research. We thank the Wuhan mental health center for helping us to organize the survey.

\section{Funding}

This research has received support from Special funds for psychological system construction in Wuhan.

\section{Availability of data and materials}

The datasets are available from the corresponding author upon reasonable request.

\section{Authors' contributions}

LXG and LWT conceptualized and designed the study. XRY and LY supervised the data collection. LXG and XRY undertook the recruitment of subjects and managed the data. LWT and LY accounted for the quality control. LY provided statistical advice on the study design and data analysis. LXG drafted the manuscript. LWT obtained funding and supervised the study. XF provided linguistic

assistance during the revision of this manuscript. All authors read and approved the final version of the manuscript.

\section{Ethics approval and consent to participate}

The ethics committee of the Wuhan mental health center approved the study, and all methods were performed in accordance with the relevant guidelines and regulations. We obtained written informed consent from all participants following clarification of the study objectives and procedure,

as well as the potential risks and benefits of participation. In addition, minors under the age of 18 years have obtained the informed consent of their legal guardian.

\section{Consent for publication}

Not applicable.

\section{Competing interests}

The authors declare that they have no competing interest.

\section{Author details}


${ }^{1}$ Research Center for Psychological Sciences and Health, China University of Geosciences, No.388, Lumo Road, Wuhan 430074, People's Republic of China.

${ }^{2}$ Zhengzhou Industry and Trade School, No.31, Yongji Road, Zhengzhou 451464, People's Republic of China.

${ }^{3}$ Wuhan Mental Hospital, Affiliated Wuhan Mental Health Center, Tongji Medical College of Huazhong University of Science \& Technology, No. 89, Gongnongbing Road, Wuhan 430012, People's Republic of China.

\section{References}

1. Pan QM. The dimension, structure and related research of adolescent aggressiveness: South China Normal University; 2005.

2. Gong YQ. Progress in psychosocial treatment of adolescent depression. Chinese School Health. $2005 ; 2: 252-3$.

3. Jiang M. Analysis of the causes of adolescent aggressive behavior and research on intervention effect: Suzhou University; 2013.

4. Lin YM. Comparison of hostile forms between patients with coronary heart disease and headache. 2000.

5. Yong N, Du L, HU H, Huang QL, Yang XY, Meng HQ. The relationship between aggressive behavior and coping style, anxiety and depression in patients with depression. Journal of the Third Military Medical University. 2014; 36:1133-7.

6. Horney K. The neurotic personality of our time: WW Norton \& Company; 1994.

7. Wu ZH, Li JM. Investigation report on the survival status of left-behind children in rural areas. Journal of China Agricultural University (Social Science Edition). 2015; 32:65-74.

8. Zhao LW. Investigation on the absence of fathers in family education and its countermeasures: East China Normal University; 2010.

9. Sun YS. Type analysis of "father absence" and its countermeasures: Shanghai Normal University; 2014.

10. Abdalla J. Hostility as a function of father absence. Derasat Nafseyah. 1992.

11. Culpin I, Heron J, Araya R, Melotti R, Joinson C. Father absence and depressive symptoms in adolescence: findings from a UK cohort. Psychological medicine. 2013; 43:2615.

12. Rosenberg M. Rosenberg self-esteem scale (RSE). Acceptance and commitment therapy Measures package. 1965; 61:18.

13. Trzesniewski KH, Donnellan MB, Robins RW. Stability of self-esteem across the life span. Journal of personality and social psychology. 2003; 84:205. 
14. Liu JX, Huang JJ, Mao FQ. Investigation on mental health problems of children with divorced parents. Chinese mental health journal. 2003; 12:846.

15. Zhang JZ. Parents' divorce must not hurt children's self-esteem -Psychological counseling cases and their analysis. Psychological World. 2004; 10:48-52.

16. Harman JP, Hansen CE, Cochran ME, Lindsey CR. Liar, liar: Internet faking but not frequency of use affects social skills, self-esteem, social anxiety, and aggression. Cyber Psychology \& Behavior. 2005; 8:1-6.

17. Zhang RJ. A study on the defensive and aggressive nature of high self-esteem under the regulation of implicit self-esteem. Jiangxi Normal University; 2005.

18. Walters AS. Using safety planning for aggressive behavior in youth: Applications to intensive treatment settings. The Brown University Child and Adolescent Behavior Letter. 2021; 3:8-8.

19. Solovjeva S, Ruzhinskikh A. The relationship of clinical and socio-psychological characteristics of hostility and aggression in patients with psoriasis. HERALD of North-Western State Medical University named after II Mechnikov. 2015; 7:113-7.

20. Bouman DE. Frustration Tolerance. 2017.

21. Berkowitz L. Frustration-aggression hypothesis: examination and reformulation. Psychological Bulletin. 1989; 106:59-73.

22. Rabinowitz S, Melamed S, Feiner M, Weisberg E, Ribak J. Hostility and hearing protection behavior: the mediating role of personal beliefs and low frustration tolerance. Journal of Occupational Health Psychology. 1996; 1:375.

23. Wu NN, Chen AY. A study on the relationship between parental rearing patterns and children's personality characteristics. Journal of Yichun university 2007(S1).

24. Xu ZY, Zu S, Xiang YT, Wang N, Guo ZH, Kilbourne AM, et al. Associations of self-esteem, dysfunctional beliefs and coping style with depression in patients with schizophrenia: A preliminary survey. Psychiatry Research. 2013; 209:340-5.

25. Ma QF, Jiang HM. Progress and significance of self-esteem research. Psychological Science. 2002; 2:242-3.

26. Deng XQ. International comparative study on the legal age of youth. Chinese youth research. 2018; 2:38-44.

27. Bai QY. The development of adolescent psychological theory and its influence on Machiavellian belief: Southwest University; 2011.

28. Xiong L, Ling H, Ye Ling. A study on the characteristics of adolescent self-concept development. Chinese Journal of Clinical Psychology. 2010; 18:511-3.

29. Zhu SZ. A study on the influence of father absence on primary school children's gender role development, self-esteem and life satisfaction: Fujian Normal University; 2011.

30. Weng JY, Lin YM, Lv BH, Chen XR, Wu YZ, Zheng YR. Development, reliability and validity test of "Short Chinese Hostility Scale". Journal of Testing. 2008; 55:463-87. 
31. Spielberger CD. State-Trait anger expression inventory. The Corsini Encyclopedia of Psychology. 2010:1.

32. Spielberger CD. The experience and expression of anger: Construction and validation of an anger expression scale. Anger and hostility in cardiovascular and behavioral disorders. 1985:5-30.

33. Xie BQ, Wu ZQ, Qin XQ. College Students' Mental Health Course. M] Wuhan: Huazhong University of Science and Technology Press .2004.34. Rosenberg M. The measurement of self-esteem, Society and the adolescent self-image. Princeton. 1965.

34. Zhou HL. Statistical Remedies for Common Method Biases. Advances in psychological science. $2004 ; 6: 942-50$.

35. Wen ZL, Ye BJ. Mediating effect analysis: development of methods and models. Advances in psychological science. 2014; 22:731-45.

36. Hayes A. Introduction to mediation, moderation, and conditional process analysis. Journal of Educational Measurement. 2013; 51:335-7.

37. Rice DR, Sullivan AD, Forehand RL, Watson $K H$, Bettis AH, Gruhn M, et al. Child Hostility toward a Parent with a History of Depression and Family Functioning. Journal of Child and Family Studies. 2020; 29:2786-95.

38. Zhao YH, Chen FZ, Zhao YD. Current situation and prospect of research on the influence of absence of fathers. International Journal of Psychiatry. 2014; 2:112-5.

39. Luo J, Wang LG, Gao WB. The influence of the absence of fathers and the timing of separation on anxiety and self-esteem of adolescents: A cross-sectional survey. Child: care, health and development. 2012; 38:723-31.

40. Shrauger JS, Rosenberg SE. Self-esteem and the effects of success and failure feedback on performance 1. Journal of personality. 1970; 38:404-17.

41. Rogers AH. The self-concept in paranoid schizophrenia. Journal of Clinical Psychology. 1958.

42. Zeng Y. Influence of absence of father on frustration and optimism of minors: Southeast China University; 2018.

\section{Figures}

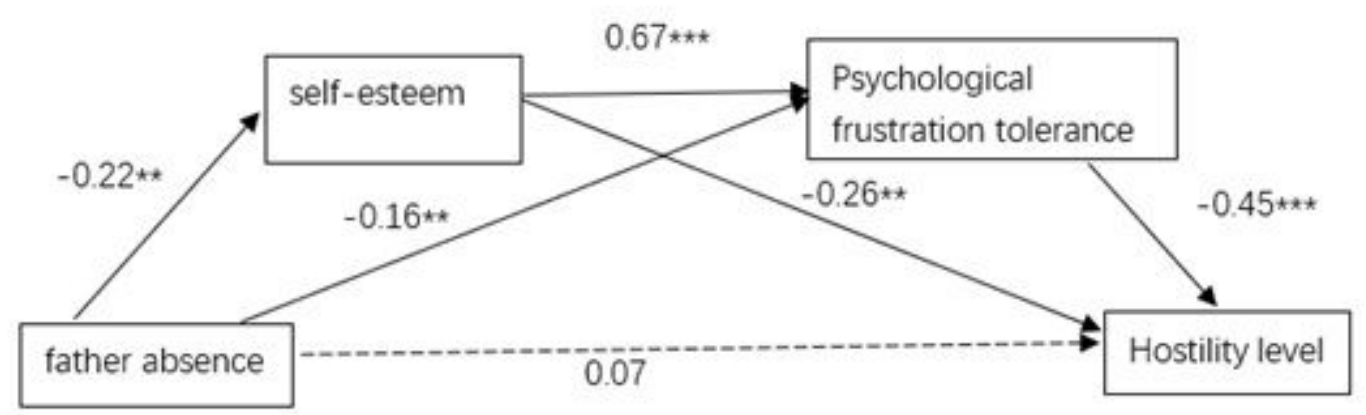


Mediation model between father absence and hostility level 